\title{
Atomic and Molecular Processes in a Strong Bicircular Laser Field
}

\author{
Dejan B. Milošević ${ }^{1,2} \mathbb{D}$ \\ 1 Faculty of Science, University of Sarajevo, Zmaja od Bosne 35, 71000 Sarajevo, Bosnia and Herzegovina; \\ milo@bih.net.ba; Tel.: +387-33-610-157 \\ 2 Academy of Sciences and Arts of Bosnia and Herzegovina, Bistrik 7, 71000 Sarajevo, Bosnia and Herzegovina
}

Received: 23 September 2018; Accepted: 5 November 2018; Published: 8 November 2018

\begin{abstract}
With the development of intense femtosecond laser sources it has become possible to study atomic and molecular processes on their own subfemtosecond time scale. Table-top setups are available that generate intense coherent radiation in the extreme ultraviolet and soft-X-ray regime which have various applications in strong-field physics and attoscience. More recently, the emphasis is moving from the generation of linearly polarized pulses using a linearly polarized driving field to the generation of more complicated elliptically polarized polychromatic ultrashort pulses. The transverse electromagnetic field oscillates in a plane perpendicular to its propagation direction. Therefore, the two dimensions of field polarization plane are available for manipulation and tailoring of these ultrashort pulses. We present a field that allows such a tailoring, the so-called bicircular field. This field is the superposition of two circularly polarized fields with different frequencies that rotate in the same plane in opposite directions. We present results for two processes in a bicircular field: High-order harmonic generation and above-threshold ionization. For a wide range of laser field intensities, we compare high-order harmonic spectra generated by bicircular fields with the spectra generated by a linearly polarized laser field. We also investigate a possibility of introducing spin into attoscience with spin-polarized electrons produced in high-order above-threshold ionization by a bicircular field.
\end{abstract}

Keywords: strong-field physics; attoscience; bicircular field; high-order harmonic generation; above-threshold ionization; spin-polarized electrons

\section{Introduction: Three-Step Model and Bicircular Laser Field}

Available strong laser fields allow the study of new laser-field-induced atomic and molecular processes such as high-order harmonic generation (HHG) [1] and above-threshold ionization (ATI) [2]. These processes are commonly considered for a laser field which is linearly polarized and explained by semiclassical three-step model [3-5]. According to this model the electron, liberated in tunnel ionization, moves driven by the laser field and returns to the parent core where it recombines emitting a high-harmonic photon in the HHG process or rescatters and is detected having much larger energy in the high-order ATI (HATI) process.

Let us explain this three-step model in more detail using Figure 1. Initially, the electron is bound with the energy $-I_{p}$. When the linearly polarized laser field $E_{\operatorname{lin}}(t)$ approaches an extremum at the time $t_{0}$, the electron can tunnel through the potential barrier, created by an instantaneous laser electric field and the atomic potential, and is "born" in the continuum with zero velocity $v\left(t_{0}\right)$. This is the first step of this model. After that, the field strength decreases, goes through zero and then reaches its next maximum value. Since the field and the corresponding force at the time $t^{\prime}$ change their signs, the electron velocity changes its direction at the time $t^{\prime \prime}$ and the electron starts moving back to its parent core. The corresponding electron velocity is related to the quantity $A(t)$, defined by 
$E_{\text {lin }}(t)=-d A(t) / d t$. Since the field $E_{\text {lin }}(t)$ is extremal at the times $t^{\prime \prime}$ and $t_{0}$ we have that $A(t)=0$ at these moments. The electron returns to the parent core at the time $t_{1}$ having the velocity $v_{\text {ret }}\left(t_{1}\right)$. This is the second step. It can be shown using momentum conservation [6], that the kinetic energy of the returned electron has the maximum value $3.17 U_{p}$, with $U_{p}=E_{\operatorname{lin}, \max }^{2} /\left(4 \omega^{2}\right)$ the electron ponderomotive energy in a linearly polarized field having frequency $\omega$ (we use atomic units). In the case of HHG process, in the third step, the electron recombines to the ground atomic state and the energy equal to ionization potential energy $I_{p}$ plus the electron kinetic energy is released in the form of an energetic photon. Maximum high-harmonic photon energy is $I_{p}+3.17 U_{p}$, as denoted in Figure 1 . The efficiency of the HHG process is approximately the same for all harmonic photons with energies larger than $I_{p}$ and the HHG spectrum has a shape of a plateau. Since the third step happens during the time interval which is a small part of the laser field optical cycle, it is clear that the described high-order atomic and molecular processes develop during few tens of attoseconds if one uses femtosecond lasers. Therefore, this third step "opens the doors" for attoscience [7-12] which investigates electron dynamics of strong-field processes on the time scale of few attoseconds, a natural scale for electronic motion in atoms and molecules (one atomic unit of time is 24.19 as).

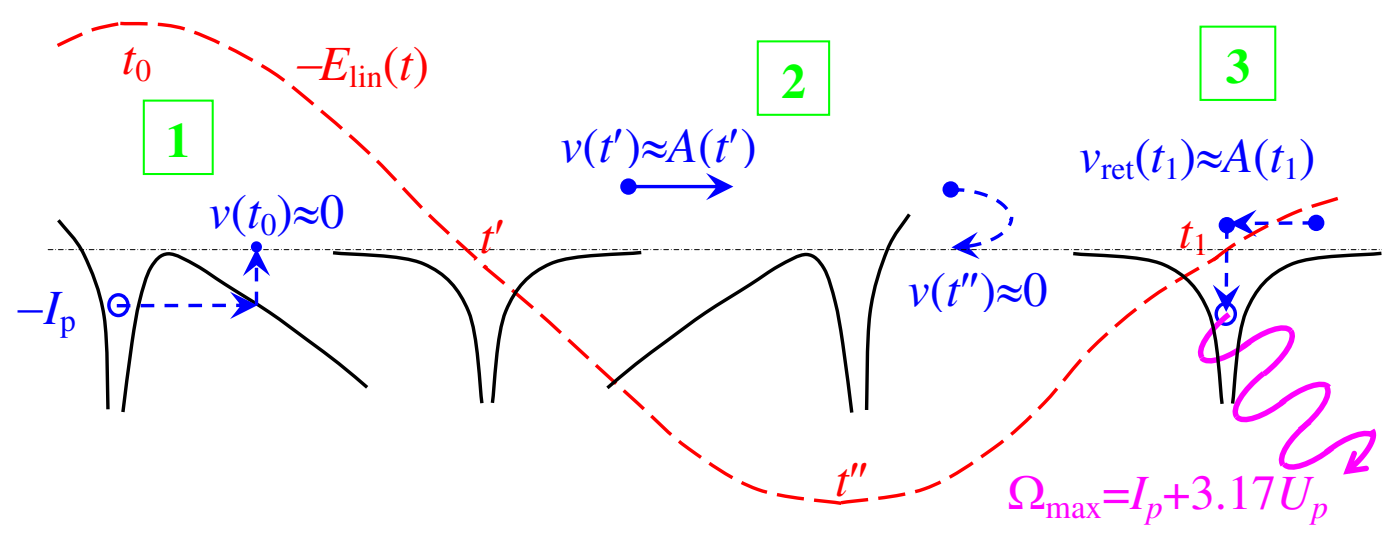

Figure 1. Graphical sketch of the three-step model for high-order harmonic generation. The combined atomic and laser field potential is presented by black lines, while the electron and its velocity are shown in blue. The temporal evolution of a linearly polarized laser field $E_{\text {lin }}(t)$ is depicted by the red long-dashed line. The emitted high-harmonic photon is illustrated by a pink wavy line with an arrow at the end.

It is well known that HATI and HHG processes are not possible with a circularly polarized laser field since the liberated electron, driven by such a field, cannot return to the parent core to recombine or rescatter. However, these processes become possible if one uses the (so-called) bicircular laser field consisting of two counter-rotating circularly polarized laser fields which are coplanar and have different frequencies. This was first confirmed experimentally for HHG in 1995 [13,14] (for more references see recent articles $[15,16])$. ATI process in a bicircular field was first investigated theoretically in $[17,18]$ (see also [19]) and confirmed experimentally in [20]. Theoretical analysis of HATI was performed in [21-23], while the relevant experimental results were published in [24,25].

In 2000 bicircular-field-induced HHG was explained using the quantum-orbit theory [26]. More information about this theory is given in [6,27]. In the present context it is important that, using this theory, two-dimensional trajectories of the electrons which come back to the parent core were identified. In addition, it was found that the emitted higher harmonics are circularly polarized with alternating ellipticities equal to \pm 1 . This was confirmed experimentally in 2014 [28]. For application it is crucial to generate circularly polarized high-order harmonics which can serve as a source of soft X-ray photons. Such photons have application for analysis of various chirality sensitive processes in organic molecules $[29,30]$, magnetic materials $[31,32]$ etc. 
Combining a group of circularly polarized high-order harmonics having ellipticities which alternate between +1 and -1 , rather than obtaining a circularly polarized pulse, we obtain a pulse having unusual polarization properties. This was first shown in [33] where, for a bicircular field with frequencies $\omega$ and $2 \omega$, a star-like form with 3 linearly polarized pulses rotated by $120^{\circ}$ was obtained. This theoretical prediction has recently been confirmed in experiment [34]. It was suggested in 2001 [35] that circularly polarized attosecond pulse trains can be generated if the harmonics having helicity +1 are stronger than that of helicity -1 (and vice versa), i.e., if we, by some means, achieve helicity asymmetry in an interval of high-harmonic photon energies. In [35] such asymmetry was noticed for He atom, which has $s$ ground state, for the intensity of the $2 \omega$ bicircular field component two times higher than that of the $\omega$ component. Later on, in 2015, it was found that the helicity asymmetry for much higher photon energies exists for HHG by noble gases with the $p$ ground state [36-38].

We study an $r \omega-s \omega$ bicircular field, with $r$ and $s$ integers, defined by

$$
\begin{aligned}
& E_{x}(t)=\left[E_{1} \sin (r \omega t)+E_{2} \sin (s \omega t+\varphi)\right] / \sqrt{2}, \\
& E_{y}(t)=\left[-E_{1} \cos (r \omega t)+E_{2} \cos (s \omega t+\varphi)\right] / \sqrt{2} .
\end{aligned}
$$

Here $I_{1}=E_{1}^{2}$ and $I_{2}=E_{2}^{2}$ are the intensities of the components and $\varphi$ is the relative phase. Examples of such fields are presented in Figure 2 for various combinations of $r$ and $s$ and the phase $\varphi=0$ (for different phases the field is rotated but does not change the shape [39]). We see that this field satisfies $(r+s)$-fold rotational symmetry. Furthermore, this field obeys particular dynamical symmetry: simultaneous rotation about the $z$ axis by the angle $r \cdot 360^{\circ} /(r+s)$ and translation in time by $T /(r+s)$ leaves the field unchanged (see the Appendix A in [38]). For example, $\omega-2 \omega$ bicircular field is invariant with respect to simultaneous rotation by $120^{\circ}$ and translation in time by $1 / 3$ optical cycle.

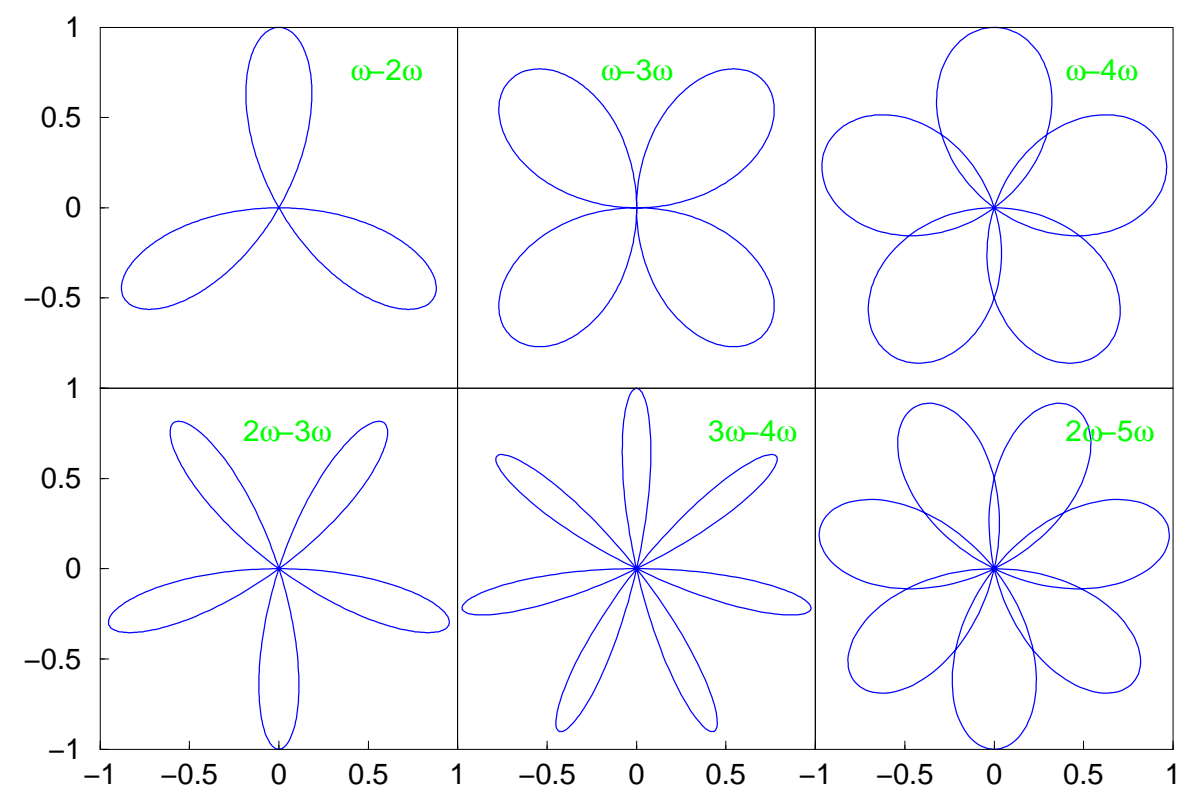

Figure 2. The electric-field-vector polar diagram for the $r \omega$-s $\omega$ bicircular field, having equal component intensities, plotted for $0 \leq t \leq T=2 \pi / \omega$, with the fundamental frequency $\omega$. The six presented panels depict the field for various combinations of the values of $r$ and $s$, as denoted. 


\section{Results for High-Order Harmonic Generation by Bicircular Field}

According to our theory of HHG by bicircular field, presented in [38], the $n$th harmonic emission rate is given by

$$
w_{n}=\frac{(n \omega)^{3}}{2 \pi c^{3}}\left|\mathbf{T}_{n}\right|^{2}, \quad \mathbf{T}_{n}=\int_{0}^{T} \frac{d t}{T} \mathbf{d}(t) e^{i n \omega t} .
$$

Here $\mathbf{d}(t)$ is the time-dependent dipole and the $n$th harmonic and its ellipticity $\varepsilon_{n}$ satisfy the following selection rule [38]

$$
\varepsilon_{n}= \pm 1 \text { for } n=q(r+s) \pm r .
$$

These relations can be derived using the dynamical symmetry of the bicircular field.

There are qualitative differences between the HHG spectrum generated by a linearly polarized laser field and the spectrum generated by bicircular field with equal component intensities. We illustrate this in Figure 3 by showing the HHG spectra, generated by Ar atoms subjected to a laser field having the fundamental photon energy $\omega=1.6 \mathrm{eV}$, as a function of the harmonic order and the laser field intensity in atomic units. For HHG by a linearly polarized laser field (upper panel (a)), emitted harmonics are linearly polarized and the spectrum forms a plateau which finishes by a cutoff. The cutoff position, i.e., the maximum harmonic order, is proportional to the laser intensity. The spectrum in the plateau exhibits fast oscillations. These oscillations are caused by the interference of the quantum-orbit contributions $[6,40,41]$. In the cutoff region there are no such oscillations since only one quantum orbit contributes to the HHG spectrum. The spectrum for $\omega-2 \omega$ bicircular field, presented in the bottom panel (b), also exhibits a plateau with a cutoff. However, the plateau is different. First, the plateau is more inclined and its height decreases with the increase of the harmonic order. Second, the plateau is flat and there are no oscillations as in the linear polarization case. The reason is that the contribution of only one quantum orbit is dominant. However, in the cutoff region there are such oscillations, again contrary to the linear polarization case. The reason is that in the cutoff region more orbits contribute to the HHG spectrum generated by bicircular laser field and the oscillatory structure is due to their interference. The relevant quantum orbits are analyzed in [26]. In the present paper we have shown, using three-dimensional graphs of Figure 3, that this behavior is valid for a wide range of laser intensities and harmonic orders. It is also clear from Figure 3 that, for a range of harmonic orders and laser intensities, the harmonic emission rate is higher for HHG by bicircular field than for HHG by linearly polarized field.

It should be mentioned that for a bicircular field with higher intensity of the second field component the plateau becomes more similar to that of the linearly polarized case, i.e., it is not inclined and oscillatory structures appear due to the interference of contributions of more quantum orbits. This is recently explored in detail in [16,42]. 

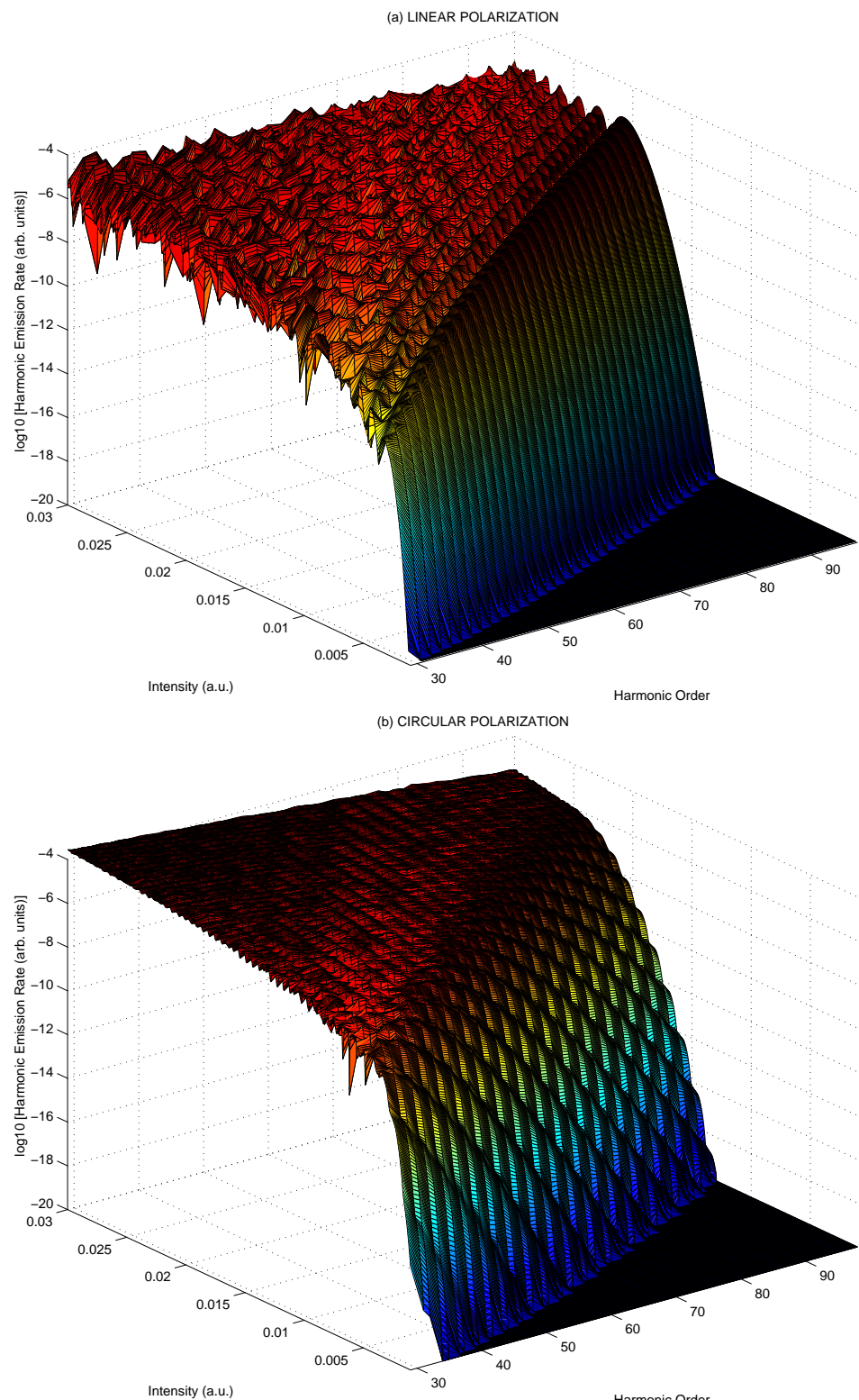

Figure 3. Three-dimensional graphs of the logarithm of the harmonic emission rate as a function of the harmonic order and laser intensity (in atomic units). Upper panel (a): Linearly polarized laser field. Lower panel (b): $\omega-2 \omega$ bicircular laser field. The fundamental photon energy is $\omega=1.6 \mathrm{eV}$ and $\mathrm{Ar}$ atoms are modeled by s ground state. The same arbitrary units for HHG rates are used in both panels.

\section{Spin Asymmetry in Above-Threshold Ionization by Bicircular Field}

In [43] (see also more recent references $[44,45]$ ) it was suggested to introduce the concept of attospin using spin-polarized electrons emitted in ionization by bicircular laser field. In this paper the differential ionization rate, $w_{\mathbf{p} \ell m}(n)=2 \pi p\left|T_{\mathbf{p} \ell m}(n)\right|^{2}$, of atoms having initial bound state $\psi_{\ell m}$, was calculated applying the saddle point-method as described in [22]. In this process the energy $n \omega$ is absorbed and an electron with momentum $\mathbf{p}$ and energy $E_{\mathbf{p}}=\mathbf{p}^{2} / 2$ is emitted. The $T$-matrix element $T_{\mathbf{p} \ell m}(n)$ is presented as a sum of the direct and rescattering $T$-matrix elements and $\ell$ and $m$ are, respectively, the orbital and magnetic quantum number. For Xe atoms the ground state is $p$ state $(\ell=1$ and $m= \pm 1$ (matrix elements are zero for $m=0)$ ), and we have two continua corresponding to two ground states of $\mathrm{Xe}^{+}$ion $\left({ }^{2} P_{3 / 2}\right.$ and $\left.{ }^{2} P_{1 / 2}\right)$. Therefore, there are two ionization potentials $I_{p}{ }^{3 / 2}=12.13 \mathrm{eV}$ and $I_{p}{ }^{1 / 2}=13.44 \mathrm{eV}$. We denote the corresponding differential ionization rates by 
$w_{\mathbf{p}, m, j}$, where $m= \pm 1$ and $j=3 / 2$ for $I_{p}{ }^{3 / 2}$ and $j=1 / 2$ for $I_{p}{ }^{1 / 2}$. For the differential ionization rate for electrons with the spin up $\left(W_{\mathbf{p} \uparrow}\right)$ and down $\left(W_{\mathbf{p} \downarrow}\right)$ we get

$$
W_{\mathbf{p} \uparrow}=\left(2 w_{\mathbf{p},-1,1 / 2}+w_{\mathbf{p},-1,3 / 2}\right) / 3+w_{\mathbf{p}, 1,3 / 2}, \quad W_{\mathbf{p} \downarrow}=\left(2 w_{\mathbf{p}, 1,1 / 2}+w_{\mathbf{p}, 1,3 / 2}\right) / 3+w_{\mathbf{p},-1,3 / 2} .
$$

We use the rates $W_{\mathbf{p} \uparrow}$ and $W_{\mathbf{p} \downarrow}$ to define the spin asymmetry parameter $A_{\mathbf{p}}$ and the normalized spin asymmetry parameter $\tilde{A}_{\mathbf{p}}$ by the relations:

$$
A_{\mathbf{p}}=\frac{W_{\mathbf{p} \uparrow}-W_{\mathbf{p} \downarrow}}{W_{\mathbf{p} \uparrow}+W_{\mathbf{p} \downarrow}}, \quad \tilde{A}_{\mathbf{p}}=A_{\mathbf{p}} \frac{W_{\mathbf{p} \uparrow}+W_{\mathbf{p} \downarrow}}{\max _{\mathbf{p}}\left(W_{\mathbf{p} \uparrow}+W_{\mathbf{p} \downarrow}\right)} .
$$

For $I_{p}{ }^{1 / 2}=I_{p}^{3 / 2}$, i.e., if we neglect the spin-orbit coupling, Formulas (4) and (5) give $A_{\mathbf{p}}=0$. In the case of Xe atoms the fine structure splitting is $I_{p}{ }^{1 / 2}-I_{p}{ }^{3 / 2}=1.31 \mathrm{eV}$ and one expects a substantial spin asymmetry. If the rates are equal for $m=1$ and $m=-1$ then the asymmetry parameter $A_{\mathbf{p}}$ is also zero. However, for ATI of noble gases having $p$ ground state by a circularly polarized laser field the ionization rate exhibits strong $m= \pm 1$ asymmetry, so that for Xe we expect large values of $A_{\mathbf{p}}$. Furthermore, for a bicircular field the electron rescattering is possible, which opens up access to attosecond spin effects, since the rescattering process develops on attosecond time scale [43].

In this paper we evaluate the ionization rate and the spin asymmetry parameter in a different way than in [43]. Namely, we calculate the differential ionization rate using numerical integration instead of the saddle-point method [46]. In addition, the results are averaged over the laser intensity distribution in the focus [47]. The used spatio-temporal averaging is applicable for long pulses, while for few-cycle pulses [8] the dynamical symmetry of the bicircular field is violated and the problem should be explored separately. From the upper left panel (a) of Figure 4 it follows that the direct differential ionization yield exhibits rotational symmetry by the angle $360^{\circ} /(r+s)=120^{\circ}$ and the reflection symmetry corresponding to the axes with angles $60^{\circ}, 180^{\circ}$, and $300^{\circ}$ with respect to the $x$ axis. The spin asymmetry parameters, shown in the left panels (middle panel (c) and bottom panel (e)), obey the same symmetry. For the rescattered electrons (right panels (b), (d), and (f)) the reflection symmetry is broken, but the rotational symmetry is maintained. The presented yields are normalized to the maximum value which is $1.237 \times 10^{-4}$ for direct electrons and $7.707 \times 10^{-5}$ for rescattered electrons (in arbitrary units since we present the results for the focal-averaged spectra). The results are normalized so that the maximum yield is $w_{\max }=1$ and $\log _{10}\left(w_{\max }\right)=0$. For the direct-electrons yields we show 6 orders, while for the rescattered-electron yields we present 4 orders of magnitude. The asymmetry parameter for direct electrons emitted in a fixed direction (for example at $60^{\circ}$ ) exhibits fast oscillations with the increase of the photoelectron energy. This was explained in [43] as the interference of two dominant electron trajectories obtained by the saddle-point method. We now see that this behaviour is preserved in the spectra obtained by numerical integration. In addition, these fast oscillations survive averaging over the laser intensity distribution in the focus. Spin asymmetry parameters change from -0.4996 to 0.98 for direct electrons and from -0.5812 to 0.8581 for rescattered electrons. The most important result is that the spin asymmetry parameter for high-energy electrons can take large values. These electrons come from rescattering and they are characterized by the attosecond time scale so that, measuring the spectra and spin-polarization of these electrons, one can explore spin-dependent effects in atoms and molecules with unprecedented time resolution. 

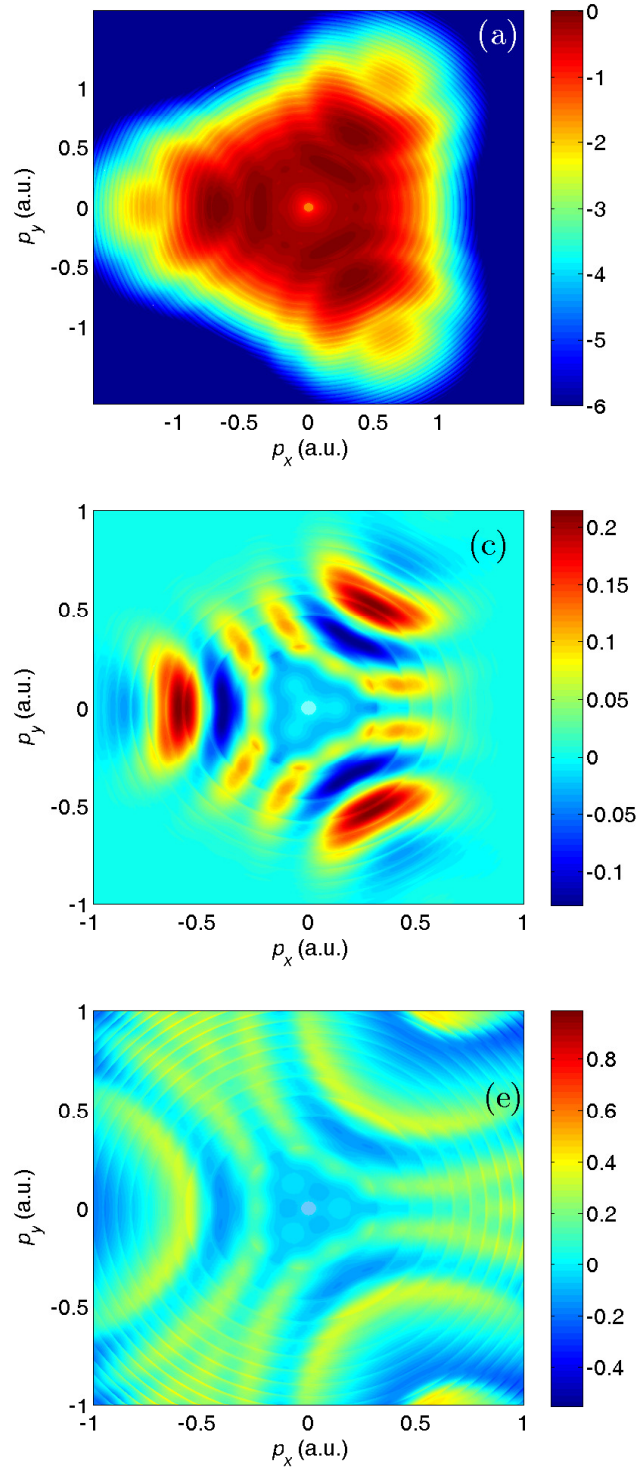
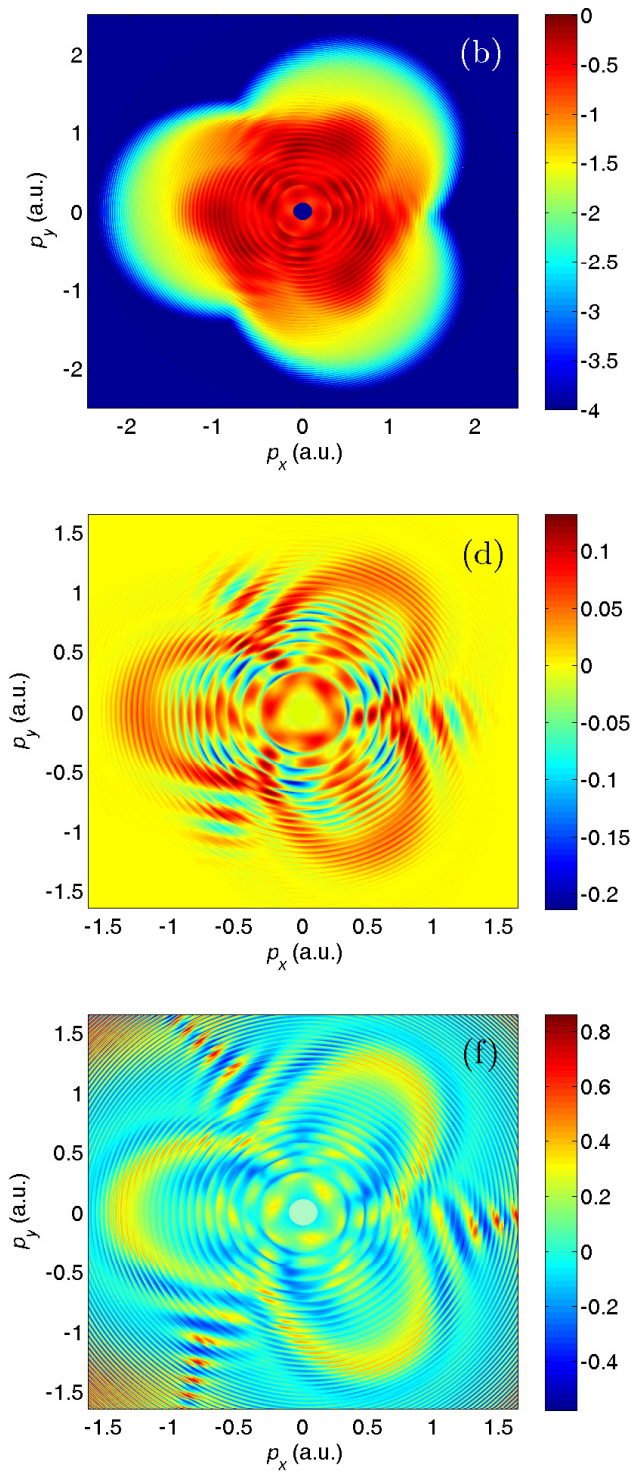

Figure 4. Focal-averaged results for Xe atoms ionized by $\omega-2 \omega$ bicircular field with the fundamental wavelength $800 \mathrm{~nm}$ and the same component peak intensities $I_{1}=I_{2}=1.1 \times 10^{14} \mathrm{~W} / \mathrm{cm}^{2}$, depicted in false colors in the photoelectron momentum plane. Top panels $(\mathbf{a}, \mathbf{b})$ : The logarithm of the summed photoelectron yield $W_{\mathbf{p} \uparrow}+W_{\mathbf{p} \downarrow}$. Middle panels (c,d): Normalized spin asymmetry parameter $\tilde{A}_{\mathbf{p}}$. Bottom panels $(\mathbf{e}, \mathbf{f})$ : Spin asymmetry parameter $A_{\mathbf{p}}$. Left panels $(\mathbf{a}, \mathbf{c}, \mathbf{e})$ : Only the direct electrons are taken into account. Right panels $(\mathbf{b}, \mathbf{d}, \mathbf{f})$ : Only the rescattered electrons are accounted for.

\section{Conclusions}

We have explored two high-order atomic processes induced by bicircular fields. First, we have explicitly compared the HHG spectra generated by a bicircular laser field with the spectra generated by a linearly polarized laser field. In spite of that both spectra exhibit plateau and cutoff features, we observed important differences. Contrary to the case of linear polarization, the plateau is rather smooth for HHG by bicircular field. This is important for obtaining a high-harmonic attosecond pulse train by combining a group of high harmonics. Namely, as in the mode-locking laser technique, the relative phase between combined field components should be constant in order to generate ultrashort pulses. This condition is much better fulfilled for HHG by bicircular field [33].

We expect that bicircular field will have a bright future in application to molecular processes. The reason is that the rotational symmetry of the $r \omega-s \omega$ bicircular field (compare Figure 2) can be 
combined with analog $C_{r+s}$ symmetry of polyatomic molecules. For example, planar molecule $\mathrm{BF}_{3}$ and nonplanar molecules $\mathrm{CF}_{3} \mathrm{I}$ and $\mathrm{NH}_{3}$ obey the $C_{3}$ symmetry, as well as the $\omega-2 \omega$ bicircular field. Examples can be found in recent references [48-53].

Another interesting possibility of application of bicircular fields is to explore the electron spin on the ultrashort time scale. Spin-polarized electrons have important applications [54,55]. Spin asymmetry in above-threshold ionization by a circularly polarized laser field was investigated theoretically in [56-58] and in more recent experiments [59-61]. In our paper we have shown that the spin asymmetry in HATI by bicircular field survives focal-averaging and thus should be observed in future experiments which will open access to attospin. It should also be mentioned that, without the focal averaging, it would not be possible to explore quantum-mechanical effects such as experimentally observed intensity-dependent enhancements in HATI spectra [2]. Such enhancements are caused by the channel-closing effect. We have recently shown that this effect is important not only for linearly polarized laser fields but also for bicircular fields [62].

In addition, the effect of the bicircular field on ATI is especially important since it can be applied to study complicated molecules and materials where the spin dependence plays an important role. For diatomic molecules it was predicted in [63] and confirmed in experiment [64] that two-source double-slit interference effects in angle-resolved HATI spectra survive both the molecular orientation averaging and focal averaging.

Funding: This research received no external funding.

Conflicts of Interest: The authors declare no conflict of interest.

\section{References}

1. Kohler, M.; Pfeifer, T.; Hatsagortsyan, K.; Keitel, C. Frontiers of Atomic High-Harmonic Generation. Adv. At. Mol. Opt. Phys. 2012, 61, 159-208. [CrossRef]

2. Becker, W.; Goreslavski, S.P.; Milošević, D.B.; Paulus, G.G. The plateau in above-threshold ionization: The keystone of rescattering physics. J. Phys. B 2018, 51, 162002. [CrossRef]

3. Corkum, P.B. Plasma perspective on strong field multiphoton ionization. Phys. Rev. Lett. 1993, 71, 1994-1997. [CrossRef] [PubMed]

4. Schafer, K.J.; Yang, B.; DiMauro, L.F.; Kulander, K.C. Above threshold ionization beyond the high harmonic cutoff. Phys. Rev. Lett. 1993, 70, 1599-1602. [CrossRef] [PubMed]

5. Paulus, G.G.; Becker, W.; Nicklich, W.; Walther, H. Rescattering effects in above-threshold ionization: A classical model. J. Phys. B 1994, 27, L703-L708. [CrossRef]

6. Becker, W.; Grasbon, F.; Kopold, R.; Milošević, D.B.; Paulus, G.G.; Walther, H. Above-threshold ionization: From classical features to quantum effects. Adv. At. Mol. Opt. Phys. 2002, 48, 35-98.

7. Scrinzi, A.; Ivanov, M.Y.; Kienberger, R.; Villeneuve, D.M. Attosecond physics. J. Phys. B 2006, 39, R1-R37. [CrossRef]

8. Milošević, D.B.; Paulus, G.G.; Bauer, D.; Becker, W. Above-threshold ionization by few-cycle pulses. J. Phys. B 2006, 39, R203-R262. [CrossRef]

9. Kling, M.F.; Vrakking, M.J.J. Attosecond electron dynamics. Annu. Rev. Phys. Chem. 2008, 59, 463-492. [CrossRef] [PubMed]

10. Krausz, F.; Ivanov, M. Attosecond physics. Rev. Mod. Phys. 2009, 81, 163-234. [CrossRef]

11. Ueda, K.; Ishikawa, K.L. Attosecond science: Attoclocks play devil's advocate. Nat. Phys. 2011, 7, 371-372. [CrossRef]

12. Calegari, F.; Sansone, G.; Stagira, S.; Vozzi, C.; Nisoli, M. Advances in attosecond science. J. Phys. B 2016, 49, 062001. [CrossRef]

13. Eichmann, H.; Egbert, A.; Nolte, S.; Momma, C.; Wellegehausen, B.; Becker, W.; Long, S.; McIver, J.K. Polarization-dependent high-order two-color mixing. Phys. Rev. A 1995, 51, R3414-R3417. [CrossRef] [PubMed]

14. Long, S.; Becker, W.; McIver, J.K. Model calculations of polarization-dependent two-color high-harmonic generation. Phys. Rev. A 1995, 52, 2262-2278. [CrossRef] [PubMed] 
15. Odžak, S.; Hasović, E.; Becker, W.; Milošević, D.B. Atomic processes in bicircular fields. J. Mod. Opt. 2017, 64, 971-980. [CrossRef]

16. Milošević, D.B. Quantum-orbit analysis of high-order harmonic generation by bicircular field. J. Mod. Opt. 2018, 66, 47-58. [CrossRef]

17. Kramo, A.; Hasović, E.; Milošević, D.B.; Becker, W. Above-threshold detachment by a two-color bicircular laser field. Laser Phys. Lett. 2007, 4, 279-286. [CrossRef]

18. Hasović, E.; Kramo, A.; Milošević, D.B. Energy- and angle-resolved photoelectron spectra of above-threshold ionizationand detachment. Eur. Phys. J. Spec. Top. 2008, 160, 205-216. [CrossRef]

19. Hasović, E.; Milošević, D.B.; Becker, W. A method of carrier-envelope phase control for few-cycle laser pulses. Laser Phys. Lett. 2006, 3, 200-204. [CrossRef]

20. Mancuso, C.A.; Hickstein, D.D.; Grychtol, P.; Knut, R.; Kfir, O.; Tong, X.M.; Dollar, F.; Zusin, D.; Gopalakrishnan, M.; Gentry, C.; et al. Strong-field ionization with two-color circularly polarized laser fields. Phys. Rev. A 2015, 91, 031402. [CrossRef]

21. Hasović, E.; Becker, W.; Milošević, D.B. Electron rescattering in a bicircular laser field. Opt. Express 2016, 24, 6413-6424. [CrossRef] [PubMed]

22. Milošević, D.B.; Becker, W. Improved strong-field approximation and quantum-orbit theory: Application to ionization by a bicircular laser field. Phys. Rev. A 2016, 93, 063418. [CrossRef]

23. Hoang, V.-H.; Le, V.-H.; Lin, C.D.; Le, A.-T. Retrieval of target structure information from laser-induced photoelectrons by few-cycle bicircular laser fields. Phys. Rev. A 2017, 95, 031402. [CrossRef]

24. Mancuso, C.A.; Hickstein, D.D.; Dorney, K.M.; Ellis, J.L.; Hasović, E.; Knut, R.; Grychtol, P.; Gentry, C.; Gopalakrishnan, M.; Zusin, D.; et al. Controlling electron-ion rescattering in two-color circularly polarized femtosecond laser fields. Phys. Rev. A 2016, 93, 053406. [CrossRef]

25. Mancuso, C.A.; Dorney, K.M.; Hickstein, D.D.; Chaloupka, J.I.; Tong, X.-M.; Ellis, J.L.; Kapteyn, H.C.; Murnane, M.M. Observation of ionization enhancement in two-color circularly polarized laser fields. Phys. Rev. A 2017, 96, 023402. [CrossRef]

26. Milošević, D.B.; Becker, W.; Kopold, R. Generation of circularly polarized high-order harmonics by two-color coplanar field mixing. Phys. Rev. A 2000, 61, 063403. [CrossRef]

27. Milošević, D.B.; Bauer, D.; Becker, W. Quantum-orbit theory of high-order atomic processes in intense laser fields. J. Mod. Opt. 2006, 53, 125-134. [CrossRef]

28. Fleischer, A.; Kfir, O.; Diskin, T.; Sidorenko, P.; Cohen, O. Spin angular momentum and tunable polarization in high-harmonic generation. Nat. Photonics 2014, 8, 543-549. [CrossRef]

29. Cireasa, R.; Boguslavskiy, A.E.; Pons, B.; Wong, M.C.H.; Descamps, D.; Petit, S.; Ruf, H.; Thiré, N.; Ferré, A.; Suarez, J.; et al. Probing molecular chirality on a sub-femtosecond timescale. Nat. Phys. 2015, 11, 654-658. [CrossRef]

30. Nahon, L.; Nag, L.; Garcia, G.A.; Myrgorodska, I.; Meierhenrich, U.; Beaulieu, S.; Wanie, V.; Blanchet, V.; Geneaux, R.; Powis, I. Determination of accurate electron chiral asymmetries in fenchone and camphor in the VUV range: Sensitivity to isomerism and enantiomeric purity. Phys. Chem. Chem. Phys. 2016, 18, 12696-12706. [CrossRef] [PubMed]

31. Fan, T.; Grychtol, P.; Knut, R.; Hernández-García, C.; Hickstein, D.D.; Zusin, D.; Gentry, C.; Dollar, F.J.; Mancuso, C.A.; Hogle, C.; et al. Bright circularly polarized soft X-ray high harmonics for X-ray magnetic circular dichroism. Proc. Natl. Acad. Sci. USA 2015, 112, 14206-14211. [CrossRef] [PubMed]

32. Kfir, O.; Zayko, S.; Nolte, C.; Sivis, M.; Möller, M.; Hebler, B.; Arekapudi, S.S.P.K.; Steil, D.; Schäfer, S.; Albrecht, M.; et al. Nanoscale magnetic imaging using circularly polarized high-harmonic radiation. Sci. Adv. 2017, 3, eaao4641. [CrossRef] [PubMed]

33. Milošević, D.B.; Becker, W. Attosecond pulse trains with unusual nonlinear polarization. Phys. Rev. A 2000, 62, 011403. [CrossRef]

34. Chen, C.; Tao, Z.; Hernández-García, C.; Matyba, P.; Carr, A.; Knut, R.; Kfir, O.; Zusin, D.; Gentry, C.; Grychtol, P.; et al. Tomographic reconstruction of circularly polarized high-harmonic fields: 3D attosecond metrology. Sci. Adv. 2016, 2, e1501333. [CrossRef] [PubMed]

35. Milošević, D.B.; Becker, W.; Kopold, R.; Sandner, W. High-Harmonic Generation by a Bichromatic Bicircular Laser Field. Laser Phys. 2001, 11, 165-168.

36. Milošević, D.B. Generation of elliptically polarized attosecond pulse trains. Opt. Lett. 2015, 40, $2381-2384$. [CrossRef] [PubMed] 
37. Medišauskas, L.; Wragg, J.; van der Hart, H.; Ivanov, M.Y. Generating isolated elliptically polarized attosecond pulses using bichromatic counterrotating circularly polarized laser fields. Phys. Rev. Lett. 2015, 115, 153001. [CrossRef] [PubMed]

38. Milošević, D.B. Circularly polarized high harmonics generated by a bicircular field from inert atomic gases in the $p$ state: A tool for exploring chirality-sensitive processes. Phys. Rev. A 2015, 92, 043827. [CrossRef]

39. Odžak, S.; Milošević, D.B. Bicircular-laser-field-assisted electron-ion radiative recombination. Phys. Rev. A 2015, 92, 053416. [CrossRef]

40. Milošević, D.B.; Becker, W. Role of long quantum orbits in high-order harmonic generation. Phys. Rev. A 2002, 66, 063417. [CrossRef]

41. Milošević, D.B.; Ehlotzky, F. Scattering and reaction processes in powerful laser field. Adv. At. Mol. Opt. Phys. 2003, 49, 373-532.

42. Milošević, D.B. Control of the helicity of high-order harmonics generated by bicircular laser fields. Phys. Rev. A 2018, 98, 033405. [CrossRef]

43. Milošević, D.B. Possibility of introducing spin into attoscience with spin-polarized electrons produced by a bichromatic circularly polarized laser field. Phys. Rev. A 2016, 93, 051402. [CrossRef]

44. Milošević, D.B. Spin-dependent effects in high-order above-threshold ionization: Spin-orbit interaction and exchange effects. J. Phys. B 2017, 50, 164003. [CrossRef]

45. Ayuso, D.; Jiménez-Galán, A.; Morales, F.; Ivanov, M.; Smirnova, O. Attosecond control of spin polarization in electron-ion recollision driven by intense tailored fields. New J. Phys. 2017, 19, 073007. [CrossRef]

46. Hasović, E.; Busuladžić, M.; Gazibegović-Busuladžić, A.; Milošević, D.B.; Becker, W. Simulation of Above-Threshold Ionization Experiments Using the Strong-Field Approximation. Laser Phys. 2007, 17, 376-389. [CrossRef]

47. Milošević, D.B.; Becker, W.; Okunishi, M.; Prümper, G.; Shimada, K.; Ueda, K. Strong-field electron spectra of rare-gas atoms in the rescattering regime: Enhanced spectral regions and a simulation of the experiment. J. Phys. B 2010, 43, 015401. [CrossRef]

48. Baykusheva, D.; Ahsan, M.S.; Lin, N.; Wörner, H.J. Bicircular High-Harmonic Spectroscopy Reveals Dynamical Symmetries of Atoms and Molecules. Phys. Rev. Lett. 2016, 116, 123001. [CrossRef] [PubMed]

49. Mauger, F.; Bandrauk, A.D.; Uzer, T. Circularly polarized molecular high harmonic generation using a bicircular laser. J. Phys. B 2016, 49, 10LT01. [CrossRef]

50. Reich, D.M.; Madsen, L.B. Illuminating Molecular Symmetries with Bicircular High-Order-Harmonic Generation. Phys. Rev. Lett. 2016, 117, 133902. [CrossRef] [PubMed]

51. Liu, X.; Zhu, X.; Li, L.; Li, Y.; Zhang, Q.; Lan, P.; Lu, P. Selection rules of high-order-harmonic generation: Symmetries of molecules and laser fields. Phys. Rev. A 2016, 94, 033410. [CrossRef]

52. Odžak, S.; Hasović, E.; Milošević, D.B. High-order harmonic generation in polyatomic molecules induced by a bicircular laser field. Phys. Rev. A 2016, 94, 033419. [CrossRef]

53. Hasović, E.; Odžak, S.; Becker, W.; Milošević, D.B. High-order harmonic generation in non-planar molecules driven by a bicircular field. Mol. Phys. 2017, 115, 1750-1757. [CrossRef]

54. Gay, T.J. Physics and technology of polarized electron scattering from atoms and molecules. Adv. At. Mol. Opt. Phys. 2009, 57, 157-247. [CrossRef]

55. Žutić, I.; Fabian, J.; Das Sarma, S. Spintronics: Fundamentals and applications. Rev. Mod. Phys. 2004, 76, 323-410. [CrossRef]

56. Barth, I.; Smirnova, O. Spin-polarized electrons produced by strong-field ionization. Phys. Rev. A 2013, 88,013401 . [CrossRef]

57. Herath, T.; Yan, L.; Lee, S.K.; Li, W. Strong-field ionization rate depends on the sign of the magnetic quantum number. Phys. Rev. Lett. 2012, 109, 043004. [CrossRef] [PubMed]

58. Barth, I.; Smirnova, O. Comparison of theory and experiment for nonadiabatic tunneling in circularly polarized fields. Phys. Rev. A 2013, 87, 065401. [CrossRef]

59. Hartung, A.; Morales, F.; Kunitski, M.; Henrichs, K.; Laucke, A.; Richter, M.; Jahnke, T.; Kalinin, A.; Schöffler, M.; Schmidt, L.P.H.; et al. Electron spin polarization in strong-field ionization of xenon atoms. Nat. Photonics 2016, 10, 526-528. [CrossRef]

60. Liu, M.-M.; Shao, Y.; Han, M.; Ge, P.; Deng, Y.; Wu, C.; Gong, Q.; Liu, Y. Energy- and Momentum-Resolved Photoelectron Spin Polarization in Multiphoton Ionization of Xe by Circularly Polarized Fields. Phys. Rev. Lett. 2018, 120, 043201. [CrossRef] [PubMed] 
61. Trabert, D.; Hartung, A.; Eckart, S.; Trinter, F.; Kalinin, A.; Schöffler, M.; Schmidt, L.P.H.; Jahnke, T.; Kunitski, M.; Dörner, R. Spin and Angular Momentum in Strong-Field Ionization. Phys. Rev. Lett. 2018, 120, 043202. [CrossRef] [PubMed]

62. Milošević, D.B.; Becker, W. Channel-closing effects in strong-field ionization by a bicircular field. J. Phys. B 2018, 51, 054001. [CrossRef]

63. Busuladžić, M.; Gazibegović-Busuladžić, A.; Milošević, D.B.; Becker, W. Angle-Resolved High-Order Above-Threshold Ionization of a Molecule: Sensitive Tool for Molecular Characterization. Phys. Rev. Lett. 2008, 100, 203003. [CrossRef] [PubMed]

64. Okunishi, M.; Itaya, R.; Shimada, K.; Prümper, G.; Ueda, K.; Busuladžić, M.; Gazibegović-Busuladžić, A.; Milošević, D.B.; Becker, W. Two-Source Double-Slit Interference in Angle-Resolved High-Energy Above-Threshold Ionization Spectra of Diatoms. Phys. Rev. Lett. 2009, 103, 043001. [CrossRef] [PubMed]

(C) 2018 by the author. Licensee MDPI, Basel, Switzerland. This article is an open access article distributed under the terms and conditions of the Creative Commons Attribution (CC BY) license (http:/ / creativecommons.org/licenses/by/4.0/). 